\title{
Antioxidant capacity and anthocyanin composition of Bulgarian bilberry (Vaccinium myrtillus L.) fruits
}

\begin{abstract}
Antioxidant capacity of Bulgarian bilberry (Vaccinium myrtillus L.) fruits from two different picking regions, Velingrad and Troyan, was studied using the FRAP (ferric reducing antioxidant power) assay and amperometric method. In addition, the anthocyanin composition was characterised by high performance liquid chromatography -diode array detection. Antioxidant capacity of the bilberries from the region of Velingrad was 15 and $20 \%$ higher than that of the Troyan region sample for the FRAP and EC $+0.5 \mathrm{~V}$ values, respectively. Among the 12 individual anthocyanins identified, delphinidin glycosides were the predominant compounds. In accordance with the content of total anthocyanins, higher concentrations $(20-64 \%)$ of individual compounds were observed for the bilberries from the region of Velingrad. The results obtained demonstrate that the environmental conditions within the picking regions have significant effects on the antioxidant capacity and anthocyanin content of Bulgarian bilberry fruits, which may affect their applications as functional foods or ingredients.
\end{abstract}

Keywords: Vaccinium myrtillus, anthocyanins, amperometric method, FRAP, HPLC
Volume 6 Issue 2 - 2018

\author{
Mariya Georgieva, ,'3 Kiril Mihalev,' Nikolina \\ Yoncheva, ${ }^{2}$ Gabriella Giovanelli ${ }^{3}$ \\ 'Department of Food Preservation and Refrigeration \\ Technology, University of Food Technologies, Bulgaria \\ ${ }^{2}$ Department of Analytical Chemistry, University of Food \\ Technologies, Bulgaria \\ ${ }^{3}$ Department of Food, Environmental and Nutritional Sciences, \\ University of Milan, Italy
}

Correspondence: Mariya Georgieva, Department of Food Preservation and Refrigeration Technology, University of Food Technologies, 26 Maritza Boulevard, 4000 Plovdiv, Bulgaria, Email inachis_io@abv.bg

Received: October 03, 2017 | Published: March 16, 2018
Abbreviations: HPLC-DAD, high performance liquid chromatography-diode array detection; FC, folin-ciocalteau's; TE, trolox equivalents; GAE, gallic acid equivalents; CGE, cyanidin 3glucoside, HPLC, high-performance liquid chromatography; FRAP, ferric reducing antioxidant power

\section{Introduction}

Epidemiological studies have shown an inverse correlation of increased fruit and vegetable consumption with a reduced risk of chronic disorders including cardiovascular diseases. Plant-derived foods contain a broad spectrum of secondary plant metabolites such as polyphenols that inhibit human low density lipoprotein oxidation, thus are made responsible for the beneficial effects on human health. ${ }^{1,2}$

Bilberry (Vaccinium myrtillus L.) is one of the most important wild berry species in Europe and is widespread in Bulgaria occurring in the semi-mountain regions at 1200-1500 meters of altitude. Among the different bioactive substances in berries, phenolic compounds including anthocyanins, flavonols, flavonols and phenolic acids have received considerable interest due to their multiple biological effects such as antioxidant, antimutagenic, antiinflammatory, antiproliferative and antimicrobial activities. ${ }^{3}$ A great number of in vitro studies showing berry polyphenols as powerful dietary antioxidants are available., The antioxidant activity is strongly dependent on the berry species, wild berries having generally higher total antioxidant capacity than the cultivated ones. ${ }^{6}$ However, extensive variations in the anthocyanin content have recently been observed for the bilberry populations, ${ }^{7}$ indicating a high degree of environmental influence. Furthermore, the proportions of specific anthocyanidins in bilberries differ between altitudes and between plants with different parental origins. ${ }^{8}$

Therefore, the present study evaluated the antioxidant capacity of Bulgarian bilberries originating from two picking regions. In addition to applying different methods to measure antioxidant capacity, the anthocyanin composition was analysed using high performance liquid chromatography-diode array detection.

\section{Materials and methods}

\section{Chemicals}

For identification and quantification purposes by high performance liquid chromatography-diode array detection (HPLC-DAD) cyanidin 3-O-glucoside, malvidin 3-O-glucoside and peonidin 3-O-glucoside (Extrasynthese, Genay, France) were used. For other analytical purposes the following reagents were used: Trolox $[( \pm)-6-$ hydroxy-2,5,7,8-tetramethyl-chroman-2-carboxylic acid] (SigmaAldrich, Steinheim, Germany); TPTZ [2,4,6-tripyridyl-s-triazine] and gallic acid monohydrate (Fluka, Buchs, Switzerland); FolinCiocalteau's (FC) reagent (Merck, Darmstadt, Germany). All other reagents and solvents were of analytical or HPLC grade.

\section{Plant material}

Bilberry (Vaccinium myrtillus L.) fruits (harvest 2010) were handpicked from their natural habitats in two different regions of Bulgaria, Velingrad and Troyan, located in the Rodopa and Stara Planina Mountain, respectively. After sorting to eliminate unripe, overripe or damaged fruits, berries were placed in polyethylene bags $(\sim 200 \mathrm{~g})$ and frozen stored $\left(-20^{\circ} \mathrm{C}, 18\right.$ months $)$ until lyophilized and transported to the laboratory in Milano.

\section{Sample preparation}

$5 \mathrm{~g}$ of the finely ground lyophilized fruits were mixed with $50 \mathrm{ml}$ of distilled water and rehydrated overnight at $4{ }^{\circ} \mathrm{C}$. The homogenate $(5 \mathrm{~g})$ was extracted with $15 \mathrm{~mL}$ of acidified methanol $(0.1 \% \mathrm{HCl}$, $\mathrm{v} / \mathrm{v}$ ) under stirring for $1 \mathrm{~h}$ in the dark at ambient temperature. After centrifugation $\left(10000 \mathrm{~g}, 10 \mathrm{~min}, 15^{\circ} \mathrm{C}\right)$, the solid phase was extracted 
for $15 \mathrm{~min}$ two more times using 15 and $10 \mathrm{~mL}$ of the extraction solvent. All supernatants were collected and the extract volume was made up to $50 \mathrm{~mL}$ with the extraction solvent. Each extraction was carried out in triplicate.

\section{Analytical methods}

Total antioxidant capacity was determined by two different methods: FRAP (ferric reducing antioxidant power) assay as described by Aaby et al. ${ }^{9}$ amperometric method as described by Buratti et al. ${ }^{10}$ This method required a flow injection system consisting of a pump (model 880 PU, Jasco, Tokyo, Japan) and an electrochemical detector (model 400, EG \& G Princeton Applied Research, Princeton, NJ) with a flow cell equipped with a double glassy carbon working electrode, an $\mathrm{Ag} / \mathrm{AgCl}$ reference electrode and a platinum counter electrode; the carrier solution was $0.1 \mathrm{~mol} / \mathrm{L}$ acetate buffer $\mathrm{pH} 4.0$ and 0.050 $\mathrm{mol} / \mathrm{L} \mathrm{NaCl}$ at a flow rate of $1 \mathrm{~mL} / \mathrm{min}$; the measurements were taken at a potential of $+0.5 \mathrm{~V}$. Trolox, a water-soluble vitamin $\mathrm{E}$ analogue, was used as a reference in both methods and the antioxidant capacity values were expressed as $\mu$ mol Trolox equivalents (TE) per $g$ of the lyophilized fruits.

Total polyphenols were determined by the Folin Ciocalteau method and expressed as mg gallic acid equivalents (GAE) per $100 \mathrm{~g}$ of the lyophilized fruits. ${ }^{11}$

Total monomeric anthocyanins were determined by the $\mathrm{pH}$ differential method. ${ }^{12}$ The results were calculated using a molar absorptivity of $26900 \mathrm{~L} /(\mathrm{mol} \mathrm{cm})$ and molecular weight of $449.2 \mathrm{~g} /$ mol and expressed as equivalents of cyanidin 3-glucoside (CGE) in $\mathrm{mg}$ per $100 \mathrm{~g}$ of the lyophilized fruits.

Identification and quantification of individual anthocyanins was performed as previously reported by Giovanelli et al. ${ }^{6}$ using a Waters HPLC system (Waters, Millford, USA) equipped with a model 600 pump, a model 2996 photodiode array detector and Empower 2 data management programme. The column used was a C18 Spherisorb ODS 2 ( $4.6 \mathrm{~mm} \times 250 \mathrm{~mm}$, Waters, Millford, USA). The mobile phase consisted of acetonitrile (eluent A) and 10\% formic acid in water (eluent B). The gradient programme was as follows: $7 \%$ to $15 \% \mathrm{~B}$ (17 $\mathrm{min}), 15 \% \mathrm{~B}$ to $30 \% \mathrm{~B}(5 \mathrm{~min}), 30 \% \mathrm{~B}$ to $100 \% \mathrm{~B}$ ( $2 \mathrm{~min}), 100 \% \mathrm{~B}$ isocratic (3 $\mathrm{min}), 100 \% \mathrm{~B}$ to $7 \% \mathrm{~B}(5 \mathrm{~min}), 7 \% \mathrm{~B}$ isocratic $(10 \mathrm{~min})$, at a flow rate of $1.2 \mathrm{~mL} / \mathrm{min}$. Monitoring was performed at $528 \mathrm{~nm}$. The injection volume was $20 \mu \mathrm{L}$. The extracts were diluted with 25 $\mathrm{mmol} / \mathrm{L} \mathrm{KCl}$ buffer $\mathrm{pH} 1.0$ before membrane filtration $(0.45 \mu \mathrm{m})$ and injection in the HPLC system. All analyses were carried out at least in triplicate.

\section{Results and discussion}

The two methods used represent different approaches for the evaluation of antioxidant capacity. While the FRAP assay quantifies the total concentration of redox-active compounds, ${ }^{13}$ the amperometric method at $+0.5 \mathrm{~V}$ has been demonstrated to selectively discriminate those compounds having effective antioxidant power. ${ }^{14-16}$

Antioxidant capacity (Table 1) of the bilberries from the region of Velingrad was 15 and $20 \%$ higher than that of the Troyan region sample for the FRAP and EC $+0.5 \mathrm{~V}$ values, respectively. This fact may be attributed to the $34 \%$ higher concentration of total anthocyanins, since the total polyphenolic contents were not significantly different. These results are in agreement with those reported in previous studies on Bulgarian bilberry fruits. ${ }^{17,18}$
As shown in Figure 1, HPLC analysis allowed identification of 12 individual anthocyanins being glucosides, galactosides and arabinosides of five anthocyanidins (delphinidin, cyanidin, peonidin, malvidin, petunidin). In general, the anthocyanin profile of the Bulgarian bilberries studied is similar to that reported for bilberry fruits from Italy, ${ }^{6}$ Finland ${ }^{7}$ and Slovenia. ${ }^{19}$ In line with these studies, delphinidin glycosides were the predominant compounds (Table 2). Derivatives of cyanidin and peonidin were found in concentrations 2.4-3.1 times lower than that of the delphinidin-derived anthocyanins and malvidin and petunidin glycosides had the lowest contents.

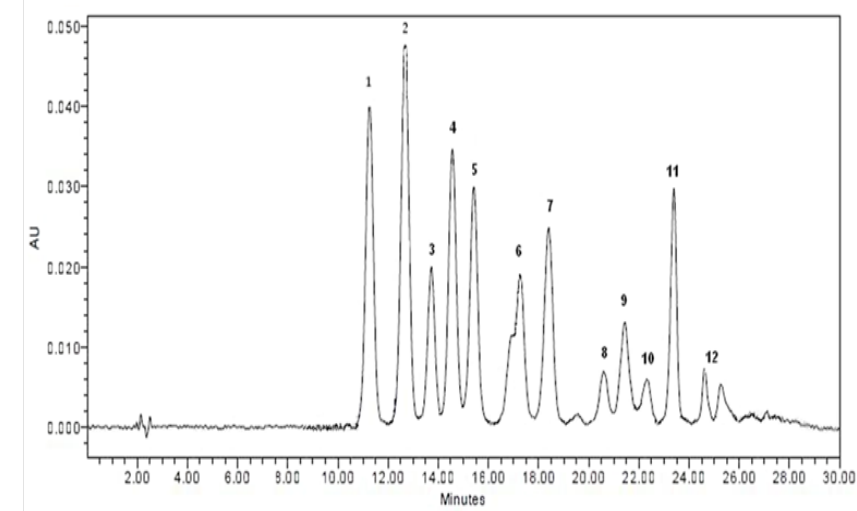

Figure I HPLC profile of anthocyanins extracted from Bulgarian bilberry fruits. Peak identification: I, delphinidin 3-galactoside; 2, delphinidin 3-glucoside; 3, cyanidin 3-galactoside; 4, delphinidin 3-arabinoside; 5, cyanidin 3-glucoside; 6, petunidin 3-galactoside+cyanidin 3-arabinoside; 7, peonidin 3-galactoside; 8, petunidin 3-arabinoside; 9, peonidin 3-glucoside; 10, malvidin 3-galactoside; I I, malvidin 3-glucoside; I2, malvidin 3-arabinoside.

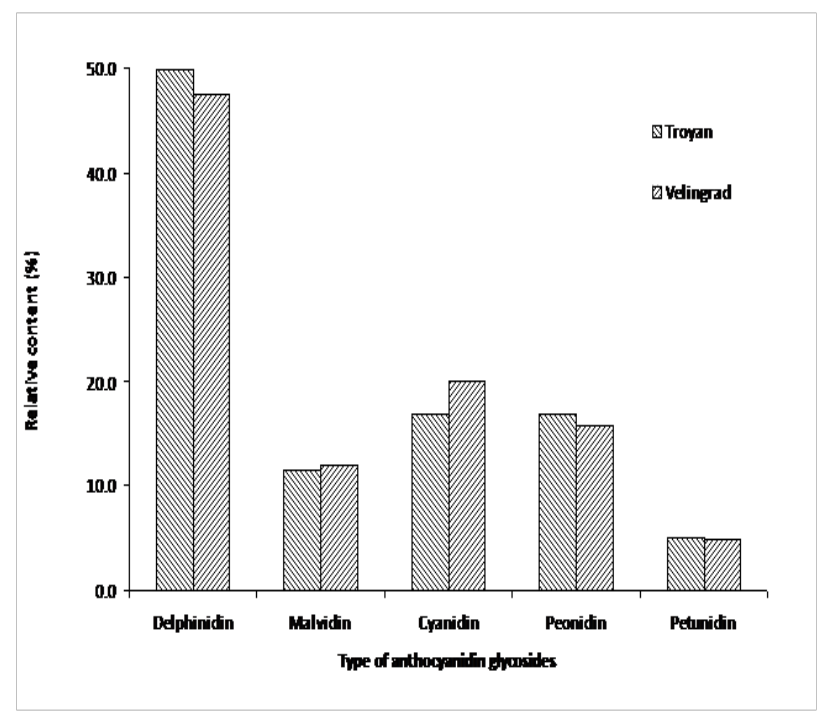

Figure 2 Proportions of anthocyanins of Bulgarian bilberry fruits from two picking regions (Troyan, Velingrad).

In accordance with the content of total anthocyanins (Table 1), higher concentrations (20-64\%) of individual compounds were observed for the bilberries from the region of Velingrad, thus suggesting the importance of environmental, e.g. climatic and edaphic, factors. Such explanation is plausible taking into account the functions of anthocyanins in plants, ${ }^{20}$ particularly to act as osmotic adjusters during periods of drought and low temperatures. The latter 
assumption is supported by results from other studies, ${ }^{7,21}$ where extensive variations in the anthocyanin contents have been reported between bilberry populations from different geographic regions. Moreover, through multivariate analysis using the contents of 27 individual polyphenols, including anthocyanins, the possibility for identification of the bilberry picking region has been recently shown. ${ }^{19}$

Table I Contents of total polyphenols and total anthocyanins and antioxidant capacity values of Bulgarian bilberry fruits from two picking regions

\begin{tabular}{|c|c|c|c|c|}
\hline \multirow[t]{2}{*}{ Picking region } & \multirow[t]{2}{*}{ Total polyphenols (mg GAE/ $100 \mathrm{~g}^{a}$ ) } & \multirow{2}{*}{$\begin{array}{l}\text { Total anthocyanins ( } \mathrm{mg} \\
\text { CGE } /\left(00 \mathrm{~g}^{\circ}\right)\end{array}$} & \multicolumn{2}{|c|}{$\begin{array}{l}\text { Antioxidant capacity } \\
\left(\mu \mathrm{molTE} / \mathrm{g}^{a}\right)\end{array}$} \\
\hline & & & FRAP & $E C+0.5 V$ \\
\hline Troyan & 893.1 & 162.4 & 2.68 & 7.26 \\
\hline Velingrad & 940.3 & 217.0 & 3.06 & 8.74 \\
\hline $\mathrm{p}$-value & 0.021 & 0.002 & $<0.001$ & $<0.001$ \\
\hline
\end{tabular}

${ }^{a}$ Values of medians are significantly different at $95 \%$ confidence level as obtained by the Mann-Whitney test (Non-parametric method).

Interestingly, some variations in the proportions of anthocyanins were observed (Figure 2); the relative content was 3\% higher and $2 \%$ lower in bilberries from the Velingrad region for cyanidin and delphinidin glycosides, respectively. These results are in accordance with the study of in which the differences observed in the proportions of bilberry anthocyanins between southern and northern Finland were supposed to be genetically determined. ${ }^{8}$ Presumably, the light and temperature as well as other factors may cause genetic differentiation in the bilberry populations occurring in different geographic regions.

Table 2 Contents ${ }^{\mathrm{a}}$ of individual anthocyanins of Bulgarian bilberry fruits from two picking regions

\begin{tabular}{|c|c|c|c|}
\hline \multirow{2}{*}{ Compound } & \multicolumn{3}{|c|}{ Picking region } \\
\hline & Troyan & Velingrad & p-value \\
\hline Delphinidin 3-galactoside ${ }^{b}$ & 924.7 & 1190.9 & $<0.001$ \\
\hline Delphinidin 3-glucoside ${ }^{b}$ & 1115.5 & 1466.5 & $<0.001$ \\
\hline Delphinidin 3-arabinoside ${ }^{b}$ & 769.5 & 909.0 & $<0.001$ \\
\hline Malvidin 3-galactoside ${ }^{c}$ & II5.I & 186.3 & $<0.001$ \\
\hline Malvidin 3-glucoside & 440.5 & 605.7 & $<0.001$ \\
\hline Malvidin 3-arabinoside ${ }^{c}$ & 81.6 & 107.2 & $<0.001$ \\
\hline Cyanidin 3-galactoside ${ }^{d}$ & 281.2 & 454.5 & $<0.001$ \\
\hline Cyanidin 3-glucoside & 676.2 & I037.I & $<0.001$ \\
\hline Peonidin 3-galactoside ${ }^{b}$ & 611.5 & 779.6 & $<0.001$ \\
\hline Peonidin 3-glucoside & 323.4 & 382.7 & $<0.001$ \\
\hline Petunidin 3-galactoside ${ }^{d}$ & 168.3 & 201.5 & $<0.001$ \\
\hline Petunidin 3-arabinoside ${ }^{d}$ & 125.2 & 150.3 & $<0.001$ \\
\hline
\end{tabular}

${ }^{\mathrm{a}}$ All data are expressed as $\mu \mathrm{mol} / \mathrm{kg}$ lyophilized fruits. Values of medians are significantly different at $95 \%$ confidence level as obtained by the Mann-Whitney test (Non-parametric method).

${ }^{\mathrm{b} C a l c u l a t e d}$ as peonidin 3-glucoside.

${ }^{\mathrm{c}}$ Calculated as malvidin 3-glucoside.

${ }^{\mathrm{d}}$ Calculated as cyanidin 3-glucoside.

\section{Conclusion}

In conclusion, the results obtained demonstrate that the environmental conditions within the picking regions have significant effects on the antioxidant capacity and anthocyanin content of Bulgarian bilberry fruits. This may affect their applications in functional food industry, especially in the light of recent ${ }^{22}$ that the effect of storage and technological processing on the content, for most of the polyphenolic classes and subclasses, is negligible in comparison to the differences between the raw materials of different plant species and/or varieties.

\section{Acknowledgement}

None.

\section{Conflict of interest}

The author declares that there is none of the conflicts.

\section{References}

1. Arts ICW, Hollman PCH. Polyphenols and disease risk in epidemiologic studies. Am J Clin Nutr. 2005;(81):317S-325S.

2. Ness AR, Powles JW. Fruit and vegetables, and cardiovascular disease: a review. Int J Epidemiol. 1997;26(1):1-13.

3. Badjakov I, Nikolova M, Gevrenova R, et al. Bioactive compounds in small fruits and their influence on human health. Biotechnology and Biotechnological Equipment. 2008;22(1):581-587.

4. Beattie J, Crozier A, Duthie GG. Potential health benefits of berries. Current Nutrition \& Food Science. 2005;1(1):71-86. 
5. Heinonen M. Antioxidant activity and antimicrobial effect of berry phenolics-a Finnish perspective. Mol Nutr Food Res. 2007;51(6):684691.

6. Giovanelli G, Buratti S. Comparison of polyphenolic composition and antioxidant activity of wild Italian blueberries and some cultivated varieties. Food Chemistry. 2009;112(4):903-908.

7. Lätti A, Riihinen K, Kainulainen P. Analysis of anthocyanin variation in wild population of bilberry (Vaccinium myrtillus L.) in Finland. J Agric Food Chem. 2008;56(1):190-196.

8. Åkerström A, Jaakola L, Bång U, et al. Effects of latitude-related factors and geographical origin on anthocyanidin concentration in fruits of Vaccinium myrtillus L. (Bilberries). J Agric Food Chem. 2010;58(22):11939-11945.

9. Aaby K, Hvattaum E, Skrede G. Analysis of flavonoids and other phenolic compounds using high-performance liquid chromatography with coulometric array detection: Relationship to antioxidant activity. $J$ Agric Food Chem. 2004;52(15):4595-4603.

10. Buratti S, Pellegrini N, Brenna OV, et al. Rapid electrochemical method for the evaluation of the oxidant power of some lipophilic food extracts. J Agric Food Chem. 2001;49(11):5136-5141.

11. Singleton VL, Rossi JA. Colorimetry of total phenolics with phosphomolybdic-phosphotungstic acid reagents. American Journal of Enology and Viticulture. 1965;(16):144-158.

12. Giusti MM, Wrolstad RE. Anthocyanins. Characterization and measurement of anthocyanins by UV-visible spectroscopy. New York: John Wiley \& Sons; 2001.

13. Magalhães LM, Segundo MA, Reis S, et al. Methodological aspects about in vitro evaluation of antioxidant properties. Analytica Chimica Acta.
2008;613(1):1-19.

14. Buratti S, Benedetti S, Cosio MS. Evaluation of the antioxidant power of honey, propolis and royal jelly by amperometric flow injection analysis. Talanta. 2007;71(3):387-1392.

15. Cosio MS, Buratti S, Mannino, S, et al. Use of an electrochemical method to evaluate the antioxidant activity of herb extracts from Labiatae family. Food Chemistry. 2006;97(4):725-731.

16. Carrasco-Pancorbo A, Cerretani L, Bendini A, et al. Evaluation of the antioxidant capacity of individual phenolic compounds in virgin olive oil. J Agric Food Chem. 2005;53(23):8918-8925.

17. Dinkova R, Georgieva M, Vasilev K, et al. Antioxidant capacity of bilberry fruits. Scientific Papers of the University of Plovdiv-Chemistry. 2011;(38):205-211.

18. Yoncheva N, Georgieva M, Ivanov G, et al. Antioxidant capacity profile of fruits from different wild berry species. Journal of Mountain Agriculture on the Balkans. 2010;(13):1475-1481.

19. Može Š, Polak T, Gašperlin L, et al. Phenolics in slovenian bilberries (Vaccinium myrtillus L.) and blueberries (Vaccinium corymbosum L.). J Agric Food Chem. 2011;59(13):6998-7004.

20. Stintzing FC, Carle R. Functional properties of anthocyanins and betalains in plants, food and in human nutrition. Trends Food Sci. Tech. 2004;15(1):19-38.

21. Burdulis D, Ivanauskas L, Dirsé V, et al. Study of diversity of anthocyanin composition in bilberry (Vaccinium myrtillus L.) fruits. Medicina(Kaunas). 2007;43(12):971-977.

22. Amarowicz R, Carle R, Dongowski G, et al. Influence of postharvest processing and storage on the content of phenolic acids and flavonoids in foods. Mol Nutr Food Res. 2009;(53):S151-S183. 